\title{
Improving Social and Communication Skills of Adult Arabs with ASD through the Use of Social Media Technologies
}

\author{
Alaa Mashat, Mike Wald, and Sarah Parsons \\ University of Southampton, UK \\ \{aam1f11, mw $@ e c s$. soton.ac.uk, \\ s.j.parsons@soton.ac.uk
}

\begin{abstract}
People with Autism Spectrum Disorder (ASD) find it hard to communicate and interact with other people. Although technology has been involved in sup-porting people with ASD in developed countries, research on such technologies has been mainly related to Western culture. Arab adults with ASD require sup-port for improving their social skills. However, cultural differences could limit the usability of existing technologies. The proposed study aims to investigate the use of social networks for supporting Arab adults with High-Functioning Autism or Asperger syndrome in order to improve their abilities in social situations such as family relations and friendships, considering the influence of culture and tradition views on the usability and sociability of social media technologies.
\end{abstract}

Keywords: Accessibility, Usability, Autism Spectrum Disorder, Arabs, Adults, Social Media, Technology, Social Skills, Communication.

\section{Introduction}

Autism Spectrum Disorder (ASD) is a lifelong developmental disability, varying from High-Functioning Autism (HFA), or Asperger syndrome, to Low-Functioning Autism (LFA) [1]. HFA and Asperger syndrome are the less severe levels of autism. People with ASD find it hard to communicate and interact with other people [2]. The National Autistic Society (UK), following DSM-5 "The Diagnostic and Statistical Manual of Mental Disorders" [3] identifies the main difficulties people with ASD face as social communication and interaction, and restricted, repetitive patterns of behaviour, interests, or activities.

In Arab countries, the situation of social communication is more difficult, as communicating with the opposite gender is already an issue with many people in certain Arab countries [4]. In addition, awareness of ASD in these countries is limited [5], and families are more likely to hide their diagnosed child to prevent themselves from feeling ashamed and from the harsh judgment of society, which could increase the isolation of people with ASD from social interaction. Many studies have been implemented with the aim of improving social skills for people with ASD; however, most research in the ASD field has been concerned with children, with only a few research 
projects on the use of technology by adults, some of which will be mentioned in Section 3. A high number of adults with ASD face problems in face-to-face communication and try to avoid social interaction situations and group activities [6,7]. About $60 \%-75 \%$ of adults with ASD have been found to have poor outcomes in their adult experiences, such as friendships, education, employment and independent living [8].

In addition, almost all of the studies and innovations have appeared in developed countries, and are mainly relevant to Western culture [9,10,11]. People with ASD receive less attention in the Arab world, and the use of technology is not widely considered. Services and support for improving their communication and interaction skills are also limited. This research is focused on investigating the use of social technologies for supporting Arab adults with High-Functioning Autism or Asperger syndrome in order to improve their abilities in social situations, considering the influence of culture and tradition on the usability of social media technologies.

\section{$2 \quad$ ASD in Arab Countries}

A number of studies have shown that the average number of diagnosed individuals in Arab countries is lower than in developed countries [9], [12,13]; however, there is also a significant difference between the numbers of ASD diagnoses in different Arab countries. This might be due to the differences in beliefs, customs and relationships or to economic problems [14]. It has been claimed by different studies [9,10], [12], that the assumption of this low percentage in countries such as Saudi Arabia, Oman and the UAE is due to the lack of information identifying children with ASD, underdiagnosis and under-reporting of cases. These point to a number of possible factors such as cultural differences, level of education, lack of services, and low levels of experience and professional training. Although, people with ASD used to receive less attention in the Arab world and fewer health care services [9], this has changed and more consideration is now given to support this group. More support centres for people with ASD have been established in different Arab countries and events and work-shops are organised to raise awareness [15,16]. However, in some Arab countries, qualified experts, and specialists are still needed in this field, in addition to support services [17]. As one of the supportive tools used in Western countries for people with ASD, technology could be used to bring its interest and usefulness to the life of people with ASD in Arab countries [18].

\section{Social Technologies and ASD}

Several studies on the use of technology have shown a potential impact on the quality of lives for people with ASD in developed countries, and the improvement of social skills [18]. Moore \& Calvert found that children with ASD enjoyed working with computer technologies, and experienced greater learning results [19]. In addition, it has been reported that users with ASD prefer to interact with technology such as computers and robots, rather than real humans because they find it safer and more interesting [20]. When it comes to socialising, observably, social networking sites have been popular in the past few. Social networks offer a type of human-human 
interaction rather than just a computer-human interaction [21], that allows users to connect and socialise without the pressures of time and immediacy that they face in real-life social situations, which could be helpful for people with ASD.

A number of studies have investigated the usability of online social networks for persons with autism spectrum conditions. These studies showed that adults with ASD could benefit from using social networks to support them in social communication, interaction, building and maintaining friendships and receiving advice. For example, a project developed by Autism Connections Europe (ACE) was presented to help adult individuals with ASD to make friends: ACEbook aims to enable friends to meet via Facebook, according to a specific interest, and then arrange personal meetings around Europe to strengthen their friendship [22]. This project has shown some improvements in social and communication skills, independence, mood, and in overcoming fear among the participants [22]. Thinking about encouraging Arab users with ASD to use a social network such as the ACEbook might raise different opinions and views due to cultural differences.

In addition, managing timing, schedules, daily life activities and self-care is also important. By working on improving these skills, people with ASD can have a better chance to be employed, be in a relationship, have friends, increase independence, have a better life and be part of the community [2]. One example of this effect comes from the Graphics, Visualization \& Usability Centre in Georgia, and is a social supportive technology for adults with ASD, implemented by Hong et al. [23]. The aim was to help adults with ASD to be more independent and to learn daily life skills, by providing them with on-demand support, with an idea similar to prompting systems. The design is called the SocialMirror; the name was driven from the idea of the device which is an interactive display integrated into a mirror and connected to an online trusted social network.

For adolescents and adults with ASD it is important to practice their social skills and learn social rules in order to improve their communication and interaction with people. In addition, a more recent study considered the use of a supportive social network aiming to provide young adults and adolescents with ASD the opportunity to gain support from a group of family and friends, instead of over-relying on one caregiver. The idea was to use circles (a feature in social networks that combines a group of people interested in a particular topic, or people with a common social connection) in order to seek information and advice, and build independence [24]. These studies showed that including social networks in assistive technologies could bring their benefits to the life of an individual with ASD.

\section{$4 \quad$ Preliminary Study}

The studies mentioned above established the usefulness of social networks for adults with ASD in Western countries. However, this may have a different perspective when considering Arab users. Based on the literature and the cultural perspective of the researcher, we have identified some cultural and traditional factors that might have an influence on Arab users with ASD when using technologies, creating a Framework for Autistic Arabs' Social Communication and Interaction Technology (FAASCIT).

Some of these factors are grouped under technical issues such as: Internet access and cost, availability of technology services, language and accents, usernames and 
passwords, variety of devices and software, cultural differences in technologies, abbreviations and Internet language and the use of Arabic language using Latin letters, which is known as "Arabizi" [25]. The other factors were grouped under personal issues, such as spoken language, accents, gender differences in the use of technology, playing music in the technology, and some related to social rules such as the restrictions towards communicating to the opposite gender, building relationships and the validity of publishing personal information and photos online. Additional factors included the level of knowledge of people with ASD and/or their parents, autonomy and independence, lack of public services and offering technologies and support.

Preliminary interviews were implemented by the researcher in the current study, aiming to gather information regarding services provided for people with ASD in Saudi Arabia, and, in addition, to find out to what extent the factors mentioned above affect the usability and accessibility of technologies by adult Arabs with ASD.

Two types of flexible, semi-structured interviews were designed, each specified for a particular group of participants: (1) a group of experts or members of staff specialized in the field of Autism, and (2) a group of adults (16 years or older) with ASD. Twelve members of staff and experts participated in this contextual review from eleven centres in different Saudi Arabian cities. Three participants were interviewed who had been diagnosed with ASD. All three were male adults aged 20, 21 and 24. However, at this stage not all the factors could be considered in the interview questions. The data collected from the interviews were in the form of text, driving the research strategy to be qualitative research.

\section{$5 \quad$ Preliminary Findings}

It was concluded that there is a lack of support for adults with ASD in Saudi Arabia and the services provided are mainly for children. Only three centres actually accept adults with ASD. One centre stops providing services at the age of 14, and one stops at the age of 16. All other centres are only for children and they do not have any facilities to support adults. One of the centres was for males only, and all other centres were for both male and female children. However, there is a segregation age at each centre at which the males and females are separated, at least in classes. The age of segregation differs between centres, ranging from 5-13 years. In addition, five of the centres do not provide Internet access to the children. Four had both Arabic and English sections, the other centres only used Arabic and did not have any English sessions. Five of the specialists reported a lack of technologies for people with ASD in the Arabic language. It was also pointed out by three of the specialists that they had faced some problems with children speaking with different accents and dialects. It was also observed that there may be some people who are not happy listening to music, but this does not really form a significant concern.

In regard to the use of social media, the three participants with ASD were not very familiar with the use of Facebook or Twitter. Two of the participants were using Whatsapp ${ }^{1}$, which "is a cross-platform mobile messaging app that allows you to exchange messages without having to pay for SMS". Even though they seemed

\footnotetext{
${ }^{1}$ http: / / www . what sapp.com/
} 
interested in computers, they only use it for basic things such as Google search, YouTube or viewing photos of people on Twitter. For presenting their personal information and pictures online, two of the participants did not have a problem; the third participant said he would not want to add his picture online. This was interesting because it was noticed that he does display his photo as a profile picture on "Whatsapp", and he had been interviewed on a T.V program before, which is now available on Youtube. In the Arabic culture, males usually do not have a problem with publishing their personal data online; this might differ with female participants.

In regard to the small number of participants, finding the right participants is challenging, as it is difficult to reach adults with HFA or Asperger syndrome in Arab countries. This could be because they may be hidden, not diagnosed or do not admit to having the disability. Widening the study to include participants from a number of other Arab countries, such as the UAE, Qatar, Kuwait and Jordan, will be considered in the further implementation of the present study.

\section{Research Strategy}

According to the Social Media Update 2013, the percentage of adults on social networking sites is $73 \%$ of online adult users, with Facebook having the highest number of users; $71 \%$ of online adults [26]. The 5th edition of the Arab Social Media Report indicates that the use of social networks has been increasing in the Arab world [27]. This significant increase in the usage of social networks could be connected to the Arab Spring, as reported in the Arab Social Media Report, [28]. In 2012 the number of Facebook users in Saudi Arabia was over six million, 90\% of whom use it in the Arabic language [29].

It has been reported in different studies that people with ASD are more interested in visuals and pictures than in text [30,31]. Putnam and Chong in [32] report that people with ASD desire to include social skills in the technologies in order to overcome their difficulties, as well as developing organisational skills and academic skills. According to Cooper [33], photos, with a percentage of about 93\%, represent the most engaging posts on Facebook, compared to links, videos or text-based posts.

For the implementation of the proposed research, the role of photos across social networks (in particular Facebook) will be investigated. The idea is to examine conversations and comments posted by Arab adults with ASD over the photos on Facebook, and how their interest in photos and their interaction can influence their social relationships with family and friends online. Participants' profiles and their activities around photos will first be evaluated. They will be given specific tasks related to photos, as part of the study, such as using tags. The activities will be evaluated after a period of time using data collected from Facebook API and face-to-face interviews.

In addition, the impact of the cultural and traditional factors during the study will be measured by creating a profile throughout the process of testing the tasks in the study, to identify the cultural factors that are important for the Arabs with ASD when using social networks, in order to develop the final framework regarding culture, and usability and sociability issues (Fig.1). 


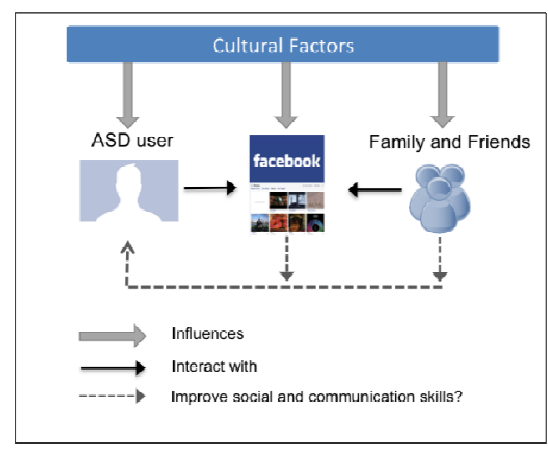

Fig. 1. Research strategy: main components and relationships

\section{Conclusion}

From the literature and the interviews, it is clear that adults with ASD in Arab countries still require more attention and support in order to improve their social and interaction skills to help them build and strengthen their relationships, and to be integrated into the society. However, Arab culture and traditions raise issues when using social networks. Our future research will investigate the effect of cultural factors on the usability and accessibility of social networks by adult Arabs with ASD, with the goal of enabling them to use the technology more easily in a way that accommodates their own cultural context and helps them to use social networks effectively for improved communication and socializing.

\section{References}

1. Newschaffer, C.J., Croen, L.A., Daniels, J., Giarelli, E., Grether, J.K., Levy, S.E., Mandell, D., Miller, L., Pinto-Martin, J., Reaven, J., Reynolds, A., Rice, C., Schendel, D., Windham, G.: The epidemiology of autism spectrum disorders*. Annu. Rev. Public Health 28, 235-258 (2007)

2. Grandin, T., Attwood, T.: Different...Not Less: Inspiring Stories of Achievement and Successful Employment from Adults With Autism, Asperger's, and ADHD: Future Horizons Incorporated (2012)

3. American Psychiatric Association, Diagnostic and Statistical Manual of Mental Disorders DSM-IV (1994)

4. Alsheikh, T., Lindley, S., Rode, J.: Understanding Online Communication through Arab Eyes. In: CHI 2010, Atlanta, Georgia, USA, April 10-15, pp. 1-4 (2010)

5. Essa, M.M., Guillemin, G.J., Waly, M.I., Al-Sharbati, M.M., Al-Farsi, Y.M., Hakkim, F.L., Ali, A., Al-Shafaee, M.S.: Increased Markers of Oxidative Stress in Autistic Children of the Sultanate of Oman. Biological Trace Element Research 147(1-3) (2012)

6. Bishop, J.: The Internet for educating individuals with social impairments. Journal of Computer Assisted Learning 19(4), 546-556 (2003)

7. Burke, M., Kraut, R., Williams, D.: Social Use of Computer-Mediated Communication by Adults on the Autism Spectrum. In: Proc. of the 2010 ACM Conference on Computer supported cooperative work (CSCW 2010), pp. 425-434. ACM, New York (2010) 
8. Seltzer, M.M., Shattuck, P., Abbeduto, L., Greenberg, J.S.: Trajectory of development in adolescents and adults with autism. Mental Retardation and Developmental Disabilities Research Reviews 10(4), 234-247 (2004)

9. Al-Farsi, Y.M., Al-Sharbati, M.M., Al-Farsi, O.A., Al-Shafaee, M.S., Brooks, D.R., Waly, M.I.: Brief Report: Prevalence of Autistic Spectrum Disorders in the Sultanate of Oman. Journal of Autism and Developmental Disorders 41(6) (2011), doi:10.1007/s10803-010-1094-8

10. Al-Salehi, S.M., Al-Hifthy, E.H., Ghaziuddin, M.: Autism in Saudi Arabia: Presentation, Clinical Correlates and Comorbidity. Transcultural Psychiatry 46(2) (2009), doi:10.1177/1363461509105823

11. Samadi, S.A., McConkey, R.: Autism in developing countries: lessons from Iran. Autism Research and Treatment (2011)

12. Eapen, V., Mabrouk, A.A., Zoubeidi, T., Yunis, F.: Prevalence of pervasive developmental disorders in preschool children in the UAE. Journal of Tropical Pediatrics 53(3) (2007), doi:10.1093/tropej/fml091

13. Yazbak, F.E.: Autism seems to be increasing worldwide, if not in London. British Medical Journal 328(7433), 226-227 (2004)

14. Hussein, H., Taha, G.R., Almanasef, A.: Characteristics of autism spectrum disorders in a sample of Egyptian and Saudi patients: transcultural cross sectional study. Child and Adolescent Psychiatry and Mental Health 5 (2011), doi:10.1186/1753-2000-5-34

15. Ashoka Arab World Internet Report: Raising autism awareness in the Arab region (2008), http: //ashokaarabworld.wordpress.com/2008/05/07/ raising-autism-awareness-in-the-arab-region/

16. Qabbani, B.: Dubai urged to step up for autism awareness' The National (2011), http: / / www . thenational ae/news / uae-news / dubai-urged-to-step-up-for-autism-awareness

17. Muhammad, F.: Saudi Gazette, Internet Report: Autism turning into regional epi-demic, experts warn (2013), http: / /www. saudigazette.com.sa/ index. cfm?method=home. regcon\&contentid=20130128150896

18. Bolte, S., Golan, O., Goodwin, M.S., Zwaigenbaum, L.: What can innovative technologies do for Autism Spectrum Disorders? Autism 14(3), 155-159 (2010)

19. Moore, M., Calvert, S.: Brief report: Vocabulary acquisition for children with autism: Teacher or computer instruction. Journal of Autism and Developmental Disorders 30(4) (2000), doi:10.1023/a:1005535602064

20. Benford, P.: The use of internet-based communication by people with autism. University of Nottingham, Nottingham (2008)

21. Spolsky, J.: It's Not Just Usability, Joel on Software (2004), http://www. joelonsoftware.com/articles/NotJustUsability.html

22. Autism Europe. Improving quality of life for people with autism (2011), http: / /www . autismeurope.org/publications / press-releases / document-497.html

23. Hong, H., Kim, J.G., Abowd, G.D., Arriaga, R.I.: Designing a social network to support the independence of young adults with autism. In: Proceedings of the ACM 2012 (2012)

24. Hong, H., Yarosh, S., Kim, J.G., Abowd, G.D., Arriaga, R.I.: Investigating the use of circles in social networks to support independence of individuals with autism. In: Paper Presented at the Proceedings of the SIGCHI Conference on Human Factors in Computing Systems. ACM (2013)

25. Ghanem, R.: Arabizi is destroying the Arabic language. Arab News (2011), http: / /www . arabnews.com/node/374897 
26. Duggan, M., Smith, A.: Social Media Update 2013. Pew Research Center (2013), http: / / pewinternet.org/Reports/2013/Social-Media-Update.aspx

27. Arab Social Media Report: Transforming Education in the Arab World: Breaking Barriers in the Age of Social Learning, 5th edn. Dubai School of Government (2013)

28. Arab Social Media Report: Social Media in the Arab World: Influencing Societal and Cultural Change? Dubai School of Government 2(1), 29 (2013)

29. The Social Clinic. The State of Social Media in Saudi Arabia 2012 (2013), http://www. thesocialclinic.com/ the-state-of-social-media-in-saudi-arabia-2012-2/

30. Habash, M.A.: Assistive Technology Utilization for Autism An Outline of Technology Awareness in Special Needs Therapy. Paper presented at the Second International Conference on Innovations in Information Technology (2005)

31. Kana, R.K., Keller, T.A., Cherkassky, V.L., Minshew, N.J., Just, M.A.: Sentence comprehension in autism: thinking in pictures with decreased functional connectivity. Brain 129(2006), 2484-2493 (2006)

32. Putnam, C., Chong, L.: Software and Technologies Designed for People with Autism: What do users want? Paper presented at the Proceedings of the 10th International ACM SIGACCESS Conference on Computers and accessibility, Canada (2008)

33. Cooper, B.B.: 7 Powerful Facebook statistics you should know for a more engaging Facebook page. buffer (2013), http: / / blog . bufferapp.com/

7-facebook-stats-you-should-know-for-a-more-engaging-page 